\title{
eRESEARCH IN THE DEGREE OF ADHERENCE BY ASTHMA PATIENTS - A PILOT STUDY
}

\author{
Anna Todorova $^{1}$, Antoaneta Tsvetkova ${ }^{2}$, Silvia Mihaylova ${ }^{3}$
}

\begin{abstract}
:
Introduction: Non-adherence to treatment is a global issue which in recent years has reached epidemic proportions. The WHO has reported that treatment adherence in chronic patients is $50 \%$ in developed countries on average. According to data from the Association of Bulgarians with Bronchial Asthma (ABBA) the percentage of asthma patients who are not regularly treated is the highest compared with other chronic diseases. Non-adherence to treatment not just influences the patient's quality of life but increases the health care costs in society.

Objectives: The aim of this study is to analyse the degree of adherence to prescribed treatment in asthma patients.

Methods: An anonymous survey was made among 50 outpatients with asthma using an adapted survey card consisting of two parts: a questionnaire that collects information about the demographic profile of the patient (gender, age, educational background, etc) and a validated tool, the Morisky test, which is a questionnaire that defines the degree of treatment adherence.

Results: The results from the Morisky test (Morisky coefficient 2.22) showed a mean degree of adherence both for the whole sample and for men and women separately, with a minimal difference of 0.01 in favour of men (Morisky coefficient 2.24 in men and 2.23 in women).

Conclusions: The studied asthma patients show unsatisfactory adherence to the prescribed treatment. The mean degree of adherence reported in the study indicates the need for educating patients, monitoring and collaboration among doctors, patients, pharmacists and other healthcare professionals.
\end{abstract}

UDC Classification: 615.1, DOI: https://doi.org/10.12955/pmp.v2.191

Keywords: asthma, adherence, Morisky test

\section{Introduction}

Bronchial asthma is characterised by a variability of symptoms and heterogeneity. Several phenotypes have been described (allergic asthma, non-allergic asthma, late-onset asthma, asthma with fixed airway obstruction and asthma in obese patients), some of which require a specific treatment regimen. No connection has been found between a specific pathological process and a particular phenotype or treatment pattern. At present, patients diagnosed with asthma in Bulgaria are treated in accordance with the stepwise approach to asthma suggested by the Global Initiative for Asthma (GINA, 2017). Better understanding of the disease mechanisms, along with technological advancements, lead to the development of new medicinal products and medical devices that allow better control of the disease. The primary treatment goal is to achieve a better control of asthma which is measured through the reduction of symptoms, maintaining normal activity levels and reduced risk of exacerbations. That can be ensured by good doctor-patient collaboration, as well as individualized control criteria for each patient consistent with their age, social, health and cultural background (GINA, 2019). Inhalation therapy is the leading therapy in the control and relief of asthma symptoms. Treatment success depends both on the doctor's choice of the right medicinal product and inhaler device and on the correct inhaler technique and patient adherence (Thomas, 2009; Foden, 2008). Despite the wide choice of proven medicinal products and variety of inhaler devices, asthma often remains uncontrolled (Small, 2011).

The level of adherence to prescribed asthma treatment both in children and adults has been extensively discussed by many authors. A search in PubMed for the keywords 'asthma, adherence' found 410 publications for 2020 only. According to Rand and Wise, the rate of non-adherence varied from $30 \%$ to $70 \%$ (Rand, 1994). The WHO has reported that in developed countries, adherence among patients suffering from chronic diseases averages $50 \%$ whereas in developing countries adherence is much lower (Sabaté, 2003).

Non-adherence is a major factor for failure in reaching the treatment goals which leads to a poor quality of life and an unfavourable disease outcome (Mäkelä, 2013). Non-adherence can be defined as

\footnotetext{
${ }^{1}$ Medical University Varna, Faculty of Pharmacy, Department of Organization and Economics of Pharmacy, Varna, Bulgaria, anna.todorova@mu-varna.bg

${ }^{2}$ Medical University Varna, Training sector "Assistant Pharmacist" Medical College, Varna, Bulgaria, antoaneta.tsvetkova@mu-varna.bg

${ }^{3}$ Medical University Varna, Training sector "Assistant Pharmacist" Medical College, Varna, Bulgaria, silviya.mihaylova@mu-varna.bg
} 
intentional and unintentional. An example of unintentional non-adherence is the incorrect use of inhaler devices due to insufficient training or lack of understanding about the inhalation technique, while refusal to take a medication is an instance of intentional non-adherence (Gillissen, 2007; Stoloff, 2004). Various methods for assessment of non-adherence in asthma patients have been developed although each of them has drawbacks. Even the current gold standard - electronic monitoring devices (EMD), have some disadvantages (Pearce and Fleming, 2018).

Objective: Study the degree of adherence to the prescribed treatment in asthma patients and contributing factors.

\section{Materials and methods}

A face-to-face anonymous survey was made among 50 outpatients with asthma. The survey was performed when the prescribed medicines were purchased in public pharmacies in Varna, the North East region of Bulgaria. In order to study the degree of adherence to the treatment anonymous surveys on paper were used. The questionnaire consisted of 10 questions, divided into two parts: the first part collected demographic information about the patient, their underlying conditions and therapeutic regimen; the second one was a validated questionnaire, a four-item Morisky scale, to assess the level of adherence to medicinal products. The score scale for the degree of adherence is presented in Table 1.

\begin{tabular}{|c|c|c|}
\hline & Score scale & Degree of adherence \\
\hline & $0-1$ & low \\
\hline & $2-3$ & medium \\
\hline & 4 & high \\
\hline
\end{tabular}

Descriptive statistics were used to determine the percentage distribution of the indicators and analysis of the results in a table. A study of the interdependences was performed using SPSS analysis. The level of statistical significance was $\mathrm{p}<0.05$.

\section{Results}

The population-based study among 50 patients in Varna, the North East region of Bulgaria showed that the most affected were people over 60 and the lowest incidence rate was in the age group 31-40. Regarding gender distribution, the percentage of women was twice that of men. The results from our survey coincided with global epidemiological data indicating that the majority of asthma patients (94\%) had one or more underlying conditions. Most of the respondents (75\%) were on a twice-daily regimen. The main characteristics of surveyed patients, regarding demographic information, underlying condition and dosage regimen are presented in Table 2.

\begin{tabular}{|c|c|c|c|}
\hline & Category & Sub-group & Distribution in \% \\
\hline & Age & $<30$ & 11 \\
\hline & & $31-40$ & 10 \\
\hline & & $41-50$ & 23 \\
\hline & & $51-60$ & 23 \\
\hline & & $>60$ & 33 \\
\hline & Gender & Male & 34 \\
\hline & & Female & 66 \\
\hline & Education & university & 41 \\
\hline & & secondary school & 37 \\
\hline & & primary school & 14 \\
\hline & Underlying conditions & yes & 94 \\
\hline & & no & 6 \\
\hline & Dosage regimen & twice a day (BID) & 75 \\
\hline & & three times a day & 19 \\
\hline & & daily $(Q D)$ & 5 \\
\hline & & $>$ three times a day & 1 \\
\hline
\end{tabular}


Adherence to the prescribed treatment is a critical factor for good control of asthma and better quality of life. There are multiple reasons for patients' non-adherence therefore a personalised approach to patients is needed (De Smet, 2006; Gaude, 2011). According to literature data factors influencing adherence are treatment regimen complexity, patient age, patient-medical professional communication, patient training and poly pharmacy (Mitkova, 2017). Table 3 provides a ranking of the reasons for nonadherence to the therapeutic regimen given by respondents.

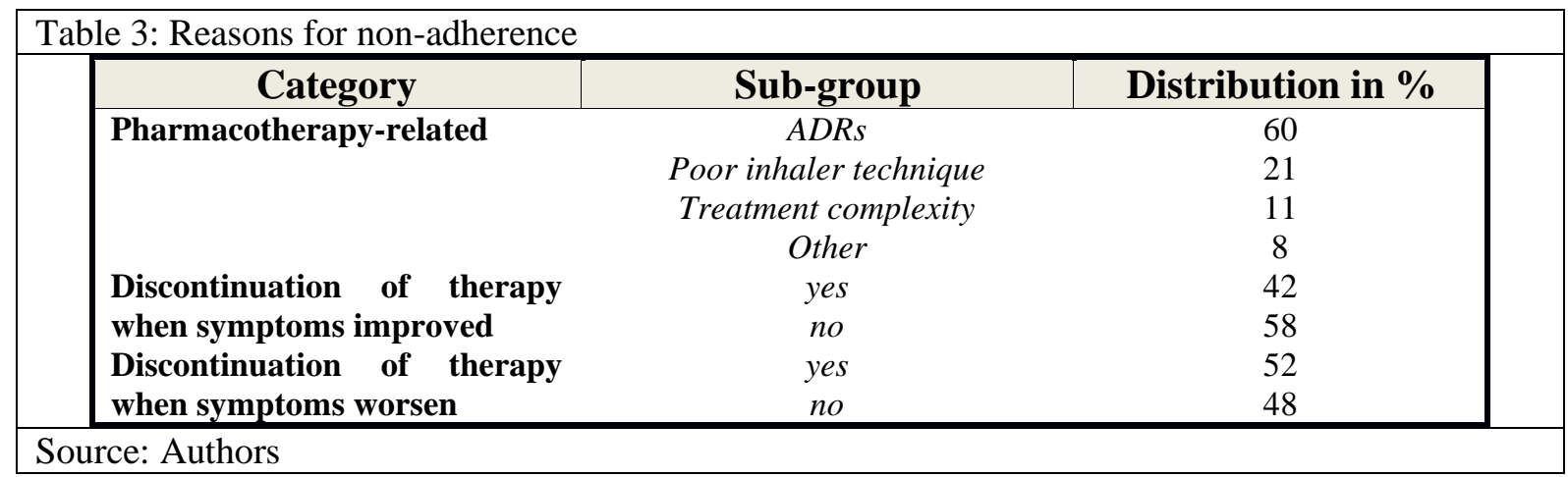

The main reason for non-adherence according to respondents (60\%) was pharmacotherapy-related issues, where $39 \%$ reported fear of possible ADRs and the remaining $21 \%$ had experienced adverse effects. A second significant reason among respondents was poor inhaler technique (21\%). Patients who consider the prescribed therapeutic regimen too complex were $11 \%$, whereas $8 \%$ reported a variety of other reasons. When symptoms improved 58\% of asthmatic patients continued with their treatment, while $42 \%$ discontinued the therapy. Our study found that more than half of the respondents $(52 \%)$ discontinued their treatment when symptoms worsened after they had followed the prescribed therapy. Disturbing results were identified regarding doctor-patient and pharmacist-patient communication. Patients tend to change their therapeutic regimen, at their own discretion, without consulting with a doctor or medical professional, either by skipping a dose or entirely discontinuing treatment.

The results from the Morisky test showed a mean degree of adherence both for the whole sample and for men and women separately, with a minimal difference of 0.01 in favour of men: the Morisky coefficient for the whole study group -2.22 , men -2.24 and women -2.23 . (Table 4)

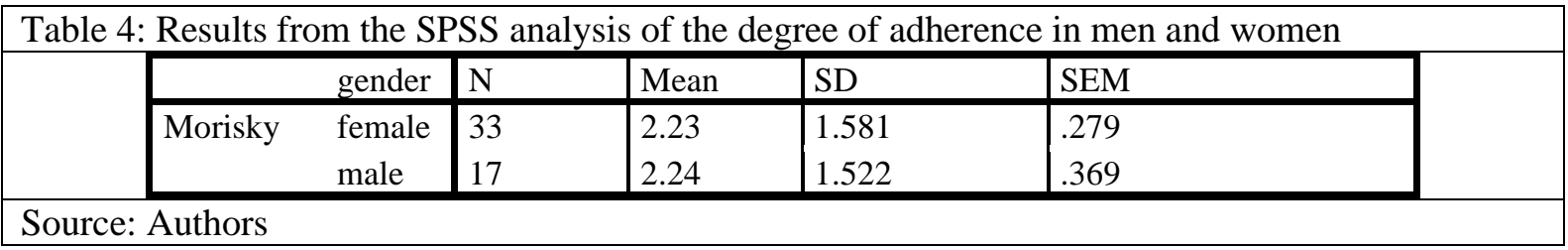

No statistically significant difference for men and women was found when studying the impact of gender on the degree of adherence $(\mathrm{p}=0.972>0.05)$. The correlation analysis of the link between age and adherence established a statistically significant inverse correlation - as age increased, adherence decreased $(\mathrm{R}=-0.304 \mathrm{p}=0.034<0.05)$. Participants with primary level of education had the highest mean value of adherence 3.40 , followed by those with a university degree 3.00 and a college degree 2.75 . The lowest degree of adherence (lowest mean value of 0.50 ) was reported by those with primary school. The correlation between the educational level and degree of adherence was studied using an ANOVA analysis. A statistical significance was determined between the level of education and the degree of adherence: the significance level of the $F$-test in ANOVA is $\mathrm{p}=0.001<0.05$. (Table 5)

\begin{tabular}{|l|l|l|l|l|l|}
\hline \multicolumn{6}{|c|}{ Table 5: Correlation between the level of education and the degree of adherence to therapy } \\
\hline & Sum of Squares & df & Mean Square & F & Sig. \\
\hline Between Groups & 39.128 & 4 & 9.782 & 5.708 & .001 \\
Within Groups & 75.402 & 44 & 1.714 & & \\
Total & 114.531 & 48 & & & \\
\hline
\end{tabular}

Source: Authors 
Analysis with the Morisky scale of the correlation between the number of daily intakes of the medicinal products and the degree of adherence showed best adherence when the intake was twice daily: Morisky coefficient 2.65. The value of the Morisky coefficient was lower when the dosage regimen was three times a day -2.13 and the lowest in the case of more than three intakes a day -0.63 .

\section{Discussion}

This study presents the demographic characteristics of asthma patients, evaluates the degree of adherence to pharmacotherapy and provides factors that influence it. Results for the studied sample of asthma patients show that $33 \%$ are over the age of $60,94 \%$ have one or more underlying conditions, as well as an unsatisfactory adherence to the prescribed therapy which corresponds with the results of other studies. Price et al. (2013) conducted a retrospective observational study of patients preferring oncedaily controller therapy for asthma and COPD. The participants were 3731 asthma patients (58\% women); the mean age was 46 years (range 2-94); of the 2138 patients with COPD, $46 \%$ were women, the mean age was 70 years (range 35-98). Approximately half of the patients in each cohort indicated once-daily preference, one-quarter were unsure, and one-quarter did not prefer once-daily therapy (Price, 2013). The mean level of adherence reported in our study indicates the need for: regular follow up of compliance by medical professionals, discussion of the prescribed therapies, patient understanding of the significance of the disease, and the conscious choice of the patient to adhere to and properly implement the therapeutic regimen. Adherence is a much more comprehensive and complex term than compliance; it does not simply mean proper use of the prescribed medicinal products, it is a multi-stage process including: compliance $\rightarrow$ adherence $\rightarrow$ concordance (Petkova, 2015). According to data from our study the factors which influence the degree of adherence are: patients' age, number of daily doses and the educational level of patients. The inverse correlations show that the adherence degree decreases with increase of age and the dosage regimen. A higher level of education is a precondition for a better adherence to the therapy. Poor adherence is mostly due to insufficient understanding of treatment goals. The main reasons identified by this study are fear of ADRs and experienced ADRs, difficulties with the inhaler technique, a treatment regimen which is too complex for patients and a tendency to arbitrarily change the dosage regimen or discontinue treatment. Inadequate inhaler technique is the result of insufficient patient training and leads to poor control of the disease (Lavorini, 2014; Newman, 2014).

Lindberg et al. (2001) attempted to identify the factors affecting compliance with the prescribed pharmacotherapy and to clarify the most important aspects of asthma care from the patient's point of view through interviews with asthma patients in primary healthcare settings in Sweden. Participants were 63 patients from 11 healthcare centres. The factors of importance for compliance were age, gender, duration of the disease, the attitude of the staff and information/education about asthma.

The most common cause of non-adherence is incorrect or poor inhaler technique in patients under the age of 65 (Foden, 2008).

It should be noted that geriatric patients are more likely to have adverse drug reactions and the fear of drug interactions is also a factor for decreased adherence. Other factors that need attention include: reduced vision (in 20\% of patients over the age of 85); motor coordination; cognitive ability; depression and isolation; co-morbidity; polypharmacy and others (Gaude, 2011).

The data collected in this survey correspond with international data both in terms of demographic indicators and in the reasons for non-adherence. The smaller number of doses is preferred by the majority of patients. Prescribed treatment patterns follow the stepwise approach suggested by GINA and available in Bulgaria. Asthma patients in Bulgaria are provided with access to the latest modern therapies.

\section{Conclusion}

Therapeutic patient education is a prerequisite for successful treatment and concordance with the treatment. Medical professionals can contribute to asthma patients' better adherence to pharmacotherapy by regularly monitoring the compliance and explaining the importance of patients' involvement and their personal role in the management and self-control of the disease. They should emphasise the seriousness of the condition, explain the action of the prescribed medicinal products, the need of their regular intake and strict adherence to the therapeutic plan. More attention has to be paid to patients' concerns regarding possible ADRs, focusing on the measures to prevent them. To avoid errors in the use of the medicine, it is necessary to demonstrate and explain in detail the inhalation technique as well as the number and sequence of doses. 


\section{References}

De Smet BD, Erickson SR, Kirking DM , et al. (2006) Self-reported adherence in patients with asthma. Ann Pharmacother. 40(3):414-20. doi: 10.1345/aph.1G475

Foden J, Hand CH. (2008) Does use of a corticosteroid/long-acting beta-agonist combination inhaler increase adherence to inhaled corticosteroids? Prim Care Respir J; 17(4):246-7 doi: 10.3132/pcrj.2008.00051

Gaude GS, (2011) Factors Affecting non-adherence in Bronchial Asthma and Impact of Health Education. Indian J Allergy Asthma Immunol.; 25(1):1-8

Gillissen, A., (2007) Patient's adherence in asthma. J Phys Pharm., 58 Suppl 5(Pt 1):205-22

GINA (2017) Global Strategy for Asthma Management and Prevention 2017 (update), available at: www.ginasthma.org

GINA (2019) Global strategy for asthma management and prevention, thttps://ginasthma.org/wp-

content/uploads/2019/06/GINA-2019-main-report-June-2019-wms.pdf

Lavorini F. (2014) Inhaled drug delivery in the hands of the patient. J Aerosol Med Pulm Drug Deliv; 27(6):414-418. doi: 10.1089/jamp.2014.1132

Lindberg M., Ekstroem T., Moeller M. et al. (2001) Asthma care and factors affecting medication compliance: the patient's point of view. International Journal for Quality in Health Care; 13(5):375-383 doi: 10.1093/intqhc/13.5.375.

Mäkelä MJ, Backer V, Hedegaard M, Larsson K. (2013) Adherence to inhaled therapies, health outcomes and costs in patients with asthma and COPD. Respir Med. 107(10):1481-90 doi: 10.1016/j.rmed.2013.04.005

Mitkova Z, Tashkov K, Dimitrova M, Manova M, (2017) Influence of the therapeutic regime on the compliance with the therapy in patients with chronic diseases in Bulgaria, Social Medicine, 2 (3); 29-32.

Morisky Medication-Taking Adherence Scale-MMAS (4-item) https://www.hal.inserm.fr/inserm-00663888/file/1472-687410-26-S2.PDF достъпено на 10.03.2021

Newman S. (2014) Improving inhaler technique, adherence to therapy and the precision of dosing: major challenges for pulmonary drug delivery. Expert Opin Drug Deliv.; 11(3):365-378. doi: 10.1517/17425247.2014.873402

Pearce C. J., Fleming L. (2018). Adherence to medication in children and adolescents with asthma: methods for monitoring and intervention. Expert review of clinical immunology; 14(12), 1055-1063 https://doi.org/10.1080/1744666X.2018.1532290

Petkova E., Popov D. (2015) Compliance and adherence to therapy in bronchial asthma. Vol. 5 (33) https://inspirobg.com/komplayans-i-pridarzhane-kam-terapiyata-pri-bronhialna-astma/, accessed on 30.03.2021

Price D., Lee A., Sims E. et al. (2013) Characteristics of patients preferring once-daily controller therapy for asthma and COPD: a retrospective cohort study. PrimCareRespirJ 22(2):161-168 doi: 10.4104/pcrj.2013.00017

Rand CS, Wise RA. (1994) Measuring adherence to asthma medication regimens. Am J Respir Crit Care Med.;149(2 Pt 2):S69-76; discussion S77-8. doi: 10.1164/ajrccm/149.2_Pt_2.S69.

Sabaté E. (2003) Adherence to Long-term Therapies: Evidence for Action. World Health Organization. Geneva; 1-194

Small M. et al., (2011) Importance of inhaler-device satisfaction in asthma treatment: Real-world observations of physicianobserved compliance and clinical/patient-reported outcomes, Advances in Therapy 28(3):202-212 doi: 10.1007/s12325-0100108-4

Stoloff SW, Stempel DA, Meyer J, Stanford RH, Carranza Rosenzweig JR. (2004) Improved refill persistence with fluticasone propionate and salmeterol in a single inhaler compared with other controller therapies. J Allergy Clin Immunol.; 113(2):245-51 DOI: 10.1016/j.jaci.2003.10.011

Thomas M, Price D, Chrystyn H, Lloyd A, Williams AE, von Ziegenweidt J. (2009) Inhaled corticosteroids for asthma: impact of practice level device switching on asthma control. BMC Pulm Med; 9:13 https://doi.org/10.1186/1471-2466-9-1 